\title{
Targeted disruption of Rab10 causes early embryonic lethality
}

\section{Dear Editor,}

Intracellular trafficking is a basis of cellular activities, including cell migration, immune response, and development (Lebreton et al., 2003; Lučin et al., 2014; Ulrich and Heisenberg, 2009). In healthy tissue, intracellular trafficking is highly regulated, controlling the form and function of cells (Furthauer and GonzalezGaitan, 2009). Work carried out in animals and plants highlights that regulated intracellular trafficking is important for the development of multicellular organisms (Fürthauer and GonzálezGaitán, 2009; Kolotuev et al., 2009; Richter et al., 2009). As a family of Ras-related small GTPase, Rabs function as coordinators for diverse aspects of intracellular trafficking including vesicle budding and formation, cargo sorting and transport to target membranes, and recruitment of key molecules (Stenmark, 2009; Zerial and McBride, 2001).

Rab10, widely distributed in intracellular membranes, is highly conserved from Caenorhabditis elegans (C. elegans) to humans. In the $C$. elegans intestine, Rab10 coordinated the transport of clathrin-independent cargo between early endosomes and recycling endosomes (Chen et al., 2006; Chen et al., 2014). Recent work in Drosophila follicle cells suggested that Rab10 is related to polarized basement membrane secretion during organ morphogenesis (Lerner et al., 2013). In mammalian systems, Rab10 has been implicated in mediating membrane targeting of plasmalemmal precursor vesicles (PPVs) during axon development (Xu et al., 2014). In addition, a large body of evidence has identify Rab10 as an essential component in basolateral recycling in Madin-Darby canine kidney (MDCK) cells and in insulin stimulated GLUT4 recycling in adipocytes (Babbey et al., 2006; Chen et al., 2012). These accumulating results strongly suggest that Rab10 is crucial and has extensive functions in cell biological processes; however, the physiological functions of Rab10 in vivo remain unclear.

In this study, we attempted to generate Rab10-deficient mice via homologous recombination in mouse embryonic stem cells to determine the contribution of Rab10 in vivo. Mouse Rab10 is located on chromosome 12 and is composed of 6 exons with the translational start codon ATG in exon 1. In our targeting strategy, we constructed a plasmid that replaced exons 3,4 , and 5 with a neomycin resistance gene cassette resulting in a frame shift in exon 6 (Fig. 1A).
The targeting plasmid was transfected into ES cells by electroporation. After screening with G418, positive ES clones were identified via PCR to amplify a $5.8 \mathrm{~kb}$ long arm and a $4.0 \mathrm{~kb}$ short arm (Fig. 1B). Four out of 102 cell clones displayed the desirable target, approximately $3.9 \%$. After confirmation using Southern blot analysis, the targeted ES clones were injected into $\mathrm{C} 57 \mathrm{BL} / 6$ blastocysts to generate chimeric mice which were backcrossed with C57BL/6 mice to produce heterozygous $\mathrm{Rab} 10^{+/-}$mice. Rab $10^{+/-}$mice were sequentially backcrossed with C57BL/6 for more than 10 generations to purify their background. Then $R a b 10^{+/-}$ mice were confirmed by Southern blot (Fig. 1C) and the offspring's genotypes were detected by PCR using genomic tail DNA as a template (Fig. 1D). Heterozygous Rab10 ${ }^{+/-}$ mice displayed no obvious abnormality in weight, diet or fertility during a 12-month observation period.

To generate Rab10-null mice, heterozygous mutants were intercrossed. Three weeks after birth, the offspring's genotypes were identified. Although $\mathrm{Rab} 10^{+/-}$parents showed no obvious abnormality, none of the 82 offspring were found to be homozygous mutants $\left(\right.$ Rab10 $\left.{ }^{-/-}\right)$(Fig. 1F) and no increased neonatal mortality was observed in the initial 3 weeks after birth. The ratio between wild-type and heterozygote mice was 0.52 , in accordance with Mendel's law. These results suggest that one functional Rab10 allele is enough for murine embryo development and adults' survival, but that a double mutant leads to embryonic lethality.

To assess the period of developmental failure, embryos from heterozygote hybridization at embryonic day 12.5 (E12.5), 10.5, and 9.5 were collected and genotyped by

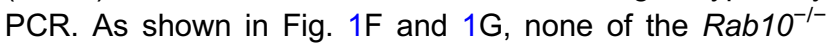
embryos was found after E9.5, suggesting that the time of embryonic lethality was before E9.5. Therefore, we continued to analyze earlier embryos at E7.5 and E8.5. In total, out of 35 E7.5 embryos, 7 (20.0\%) were homozygous mutants and $1(2.9 \%)$ was a resorbed embryo; out of $71 \mathrm{E} 8.5 \mathrm{em}$ bryos, $8(11.3 \%)$ were homozygous mutants and $10(14.1 \%)$ were resorbed embryos (Fig. 1G). To further confirm these results, E7.5 embryos were collected and analyzed by Western blot with anti-Rab10 antibody. As shown in Fig. 1E, Rab10 protein was totally deleted in Rab10-null mice. $R a b 10^{-1-}$ mutants showed obvious differences from wildtype embryos (Fig. 1I). Mutant embryos at E7.5 were much 
A
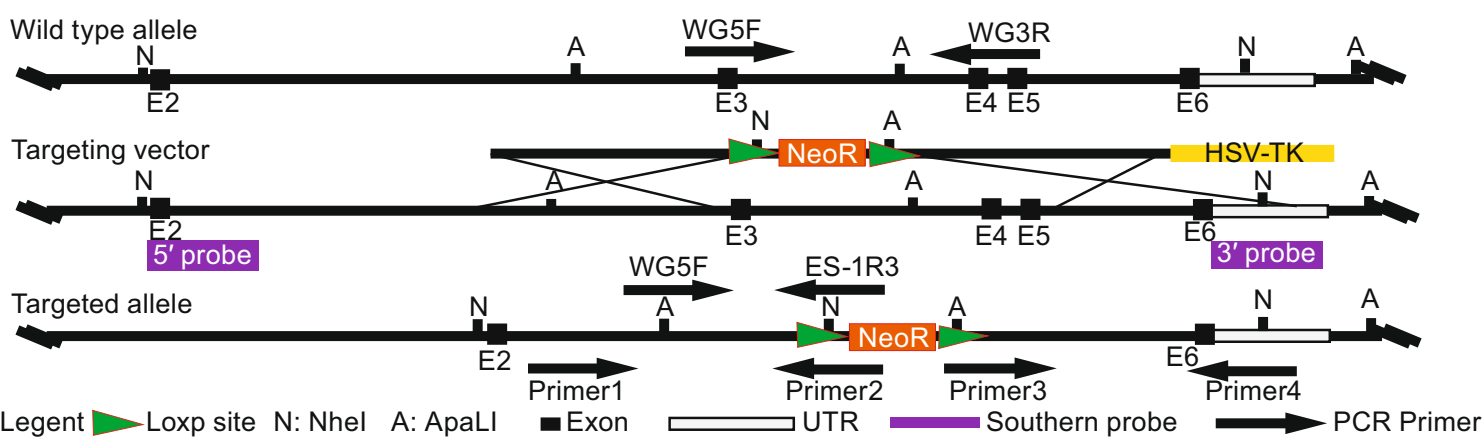

B

F

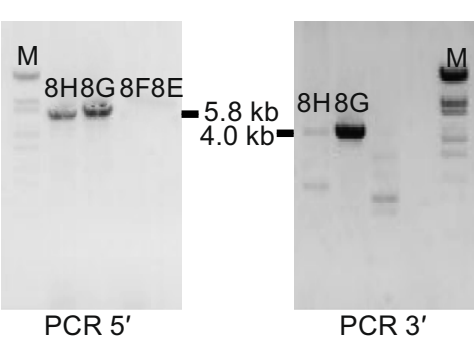

\begin{tabular}{|c|c|c|c|c|}
\hline \multirow{2}{*}{ Age } & \multicolumn{3}{|c|}{ No. of mice with genotype } & \multirow{2}{*}{ Total } \\
\cline { 2 - 4 } & $+/+$ & $+/-$ & $-/-$ & \\
\hline E10.5 & 9 & 23 & 0 & 32 \\
\hline E12.5 & 10 & 19 & 0 & 29 \\
\hline 3 weeks & 28 & 54 & 0 & 82 \\
\hline
\end{tabular}

$\mathrm{H}$
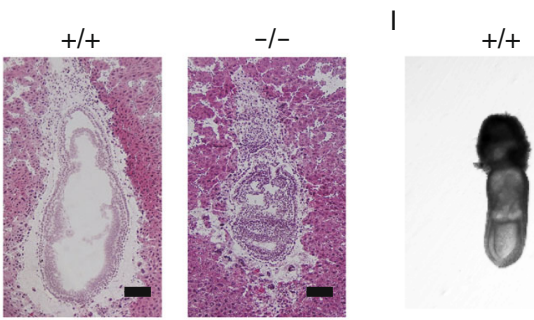

C

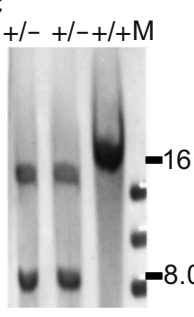

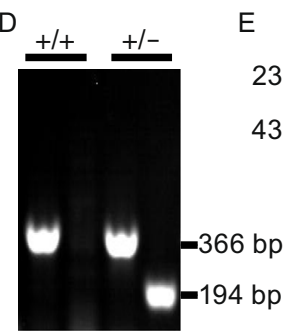

E

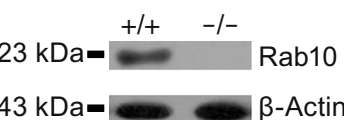

G

\begin{tabular}{|c|c|c|c|c|}
\hline \multirow{2}{*}{ Age } & \multicolumn{3}{|c|}{ No. of mice with genotype } & \multirow{2}{*}{ Total } \\
\cline { 2 - 4 } & $+/+,+/-$ & $-/-$ & Resorbed & \\
\hline E7.5 & 27 & 7 & 1 & 35 \\
\hline E8.5 & 53 & 8 & 10 & 71 \\
\hline E9.5 & 22 & 0 & 5 & 27 \\
\hline
\end{tabular}

+/+

$-1-$

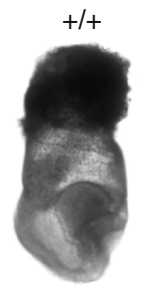

E8.5

E7.5

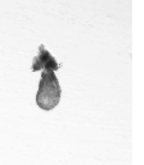

5

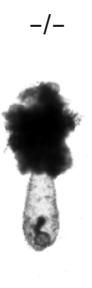

Figure 1. Rab10 deficiency causes early embryonic lethality. (A) Schematic representation of the Rab10 locus, the targeting vector, and the targeted locus. The black boxes represent exon 2-6 of Rab10 gene. Arrows show the primers used for PCR analysis. (B) Left: PCR analysis to detect 5' recombination in transfected ES cells with primers 1 and 2. Right: PCR analysis to detect $3^{\prime}$

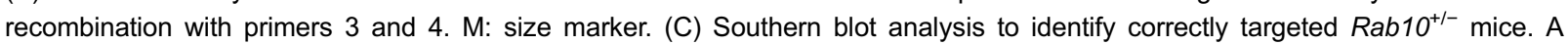

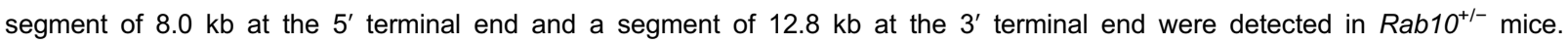
(D) Genotyping of $R a b 10^{+/+}$and $R a b 10^{+/-}$mice by PCR analysis. (E) Rab10 level was analyzed by Western blot in Rab10 ${ }^{+/+}$and Rab10 $0^{-1-}$ embryos. (F) Genotypes of progeny aged E10.5, E12.5 and 3 weeks after birth from Rab10 heterozygote intercrosses. (G) $\mathrm{Rab}^{-/-}$embryos from intercrosses of heterozygous littermates recovered at E7.5. $(\mathrm{H})$ Histological analysis of E7.5 embryos developing in the uterus. Typical pictures of wild-type and Rab10 knockout embryos are displayed in the left and right panels, respectively. Scale bar $=100 \mu \mathrm{m}$. (I) Appearance of Rab10-deficient embryos from E7.5 to E8.5 and totally resorbed at E9.5.

smaller and the development of the three layers (endoderm, mesoderm, and ectoderm) was largely arrested. Hematoxylin and eosin staining of histological sections of E7.5 are shown in Fig. 1H. Extraembryonic ectoderm of Rab10 knockout embryos was not well organized and primitive streak formation was not observed in the embryonic region.
The ectoplacental cavity, exocoelomic cavity, and amniotic cavity were abnormal. At E8.5, the embryos had degenerated much more seriously, with very little to almost no identifiable structure. Along with embryonic degeneration, the yolk sac was greatly degraded. Consequently, the abnormal embryos were totally absorbed at E9.5. Taken 
together, these data demonstrate that the development of Rab10 ${ }^{-1-}$ embryos is defective approximately at E7.5 and the mutant embryos are totally resorbed at E9.5.

Based on our observation of E7.5 developmental failure above, we used an immunofluorescent assay with Ki67 (Abcam) and 4',6-diamidino-2-phenylindole (DAPI) staining to determine whether defective development of Rab10 ${ }^{-1-}$ embryos was due to the failure of cell proliferation. The embryos were separated at E7.5 after fertilization to obtain $10 \mu \mathrm{m}$ frozen sections for immunofluorescence. As shown in Fig. 2A and 2C, approximately $89 \%$ of the cells were Ki67positive in wild-type embryos; however, only $60 \%$ of the cells were Ki67-positive in mutant embryos, suggesting a dramatic reduction in cellular proliferation.

None of the Rab10-null embryos grew after E7.5, and many of the cells were proliferative-inactive. To determine the reasons underlying the massive cell death in mutant conceptuses and eventual embryo destruction, terminal deoxynucleotidyl transferase-mediated dUTP-biotin nick end labeling (TUNEL) staining was used to detect the ratio of apoptotic cells in wild-type and Rab10 ${ }^{-1-}$ embryos (Fig. 2B). Embryonic frozen sections were stained with DAPI to label cell location and fluorescence microscopy was used to record $405 \mathrm{~nm}$ and $561 \mathrm{~nm}$ at the same time. Statistical analysis revealed that few TUNEL-positive cells were de-

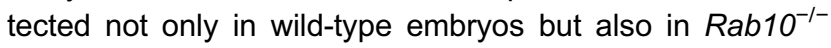
littermates, suggesting that Rab10-null embryos did not undergo significant apoptosis at E7.5.

When we analyzed the size of nucleus, however, we noticed that the nuclei diameter in the $\mathrm{Rab}_{10^{-/-}}$mutants was reduced by $13 \%$ compared with that of the wild-type embryos. The diameter of the nuclei in wild-type embryos was $11.9 \mu \mathrm{m}$, whereas in Rab10-null mice, the diameter was $10.3 \mu \mathrm{m}$ (Fig. 2D and 2E). Because of the critical role of the nucleus during cellular activities such as cell division, mRNA transcription and protein expression, the variation of nuclear size may be an indication of other significant cellular changes.

Because of the diminished number of cells and the decreased size of the nucleus in $R a b 10^{-1-}$ embryos, we hypothesized that morphological changes in subcellular structures may be present in the Rab10 mutants. It has been reported that in $C$. elegance, Rab10 mutation caused enlarged vacuoles of early endosome origin by blocking export from the basolateral early endosomes (Chen et al., 2006). Because the Rab10 gene is highly conserved between different species, we suspected that $\mathrm{Rab}^{-10^{-/}}$cells may show a similar phenotype. We analyzed histological sections using immunofluorescence to label different organelles followed by confocal microscopy; however, structural details could not be observed clearly (data not shown) because the large scale of the nucleus occupied most of the cytoplasmic space and the resolution was limited. We then used transmission electron

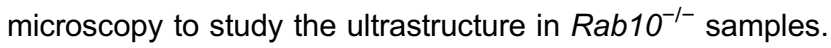
As shown in Fig. 2F, the cytoplasm was very dense and full of ribosomes in wild-type cells, which reflected the high synthetic activities of proteins. On the contrary, in Rab10 ${ }^{-/-}$ cells, many vacuoles were accumulated in the cytoplasm (Fig. 2F and 2G). The size of these vacuoles varied from $400 \mathrm{~nm}$ to $1600 \mathrm{~nm}$. Moreover, the ER displayed hyperplasia compared with wild-type embryos (Fig. 2H). Considering these results, loss of Rab10 function results in abnormal endosomes and ER hyperplasia, likely disturbing the balance of membrane trafficking and ultimately leading to the death of the embryos.

In summary, we presented the study of Rab10 function in conventional knockout mice and demonstrated that Rab10 executes critical and non-redundant functions in the early development of mammalian embryos. First, no $\mathrm{Rab}_{10^{-1-}}$ mice or embryos were found after E9.5, and approximately $14.1 \%$ of embryos at E8.5 were completely resorbed. At E7.5, all of the abnormal embryos were $R a b 10^{-1-}$. Second, the dramatic reduction of Ki67 positive cells in Rab10-null embryos, followed by cell death, suggested that a defect in cellular proliferation underlies the developmental failure.

Rab10 has been reported to localize in many organelles, such as ER, golgi, early endosome, recycling endosome, and mediates membrane trafficking within the cell, which counterbalances secretion and permits complex interplay between cells and their environment. Although the Rab10deficient embryos' growth ceased at E7.5 followed by resorption at E8.5, massive cell death did not result from apoptosis (Fig. 2B). RAB10 deficiency results in the accumulation of abnormally abundant RAB-5-positive vacuoles and it is regarded as a key regulator of endocytic recycling in polarized epithelial cells of the $C$. elegans intestine (Chen et al., 2006). In this study, we successfully observed this phenomenon in Rab10 mutant embryos. Vacuoles of different sizes, presumably early endosomes, were accumulated in the cytoplasm of Rab10-defective cells, which indicated the endosomal trafficking was largely defected in $R a b 10^{-1-}$ cells. The eukaryotic endosomal trafficking is essential for the internalization and trafficking of macromolecules, fluid, membranes, and membrane proteins. The disruption of endosomal trafficking that leads to a cellular dysfunction may be responsible for the death of $R a b 10^{-/-}$embryos.

In the development of mouse embryos, gastrulation is initiated in the epiblast at about E6.5 and three germ layers are well established at around E7.5. At E8.0, somites first appear and heart starts to form. Given that the developmental disruption of Rab10-null embryos occurred at approximately E7.5, we suspected Rab10 should function earlier before E7.5 in the development of mice embryos. However, the exact mechanism about why Rab10 deficiency results in early embryonic cell death and tissue abnormalities needs to be further characterized.

This work is the first attempt to access the physiological function of Rab10 protein using a Rab10 conventional knockout mouse model. Here we clearly demonstrate that Rab10 is essential for early embryogenesis. However, as the Rab10 mutation in mice was difficult to study due to the 

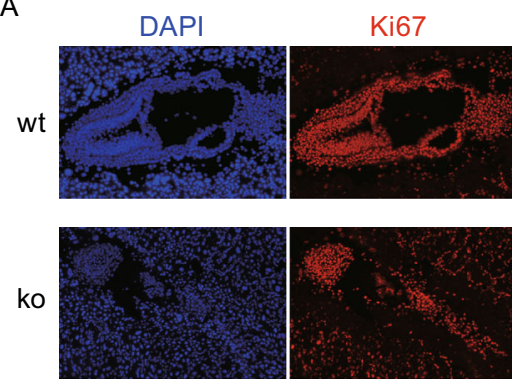

C

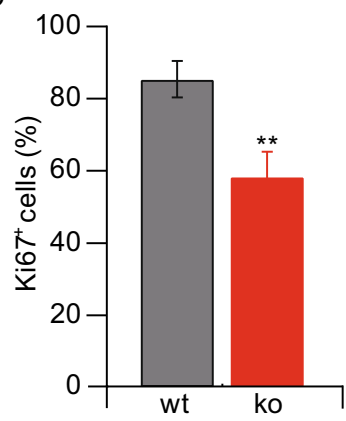

D

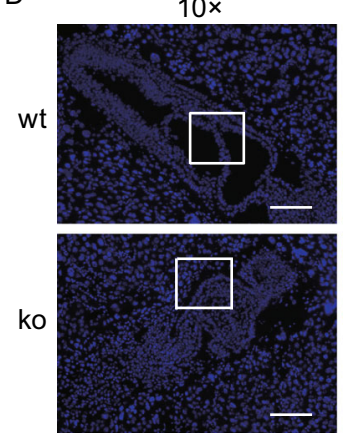

F
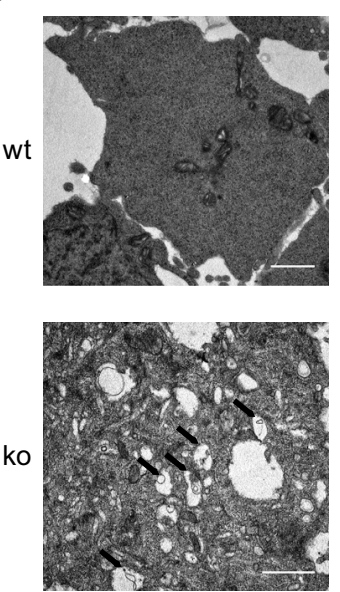
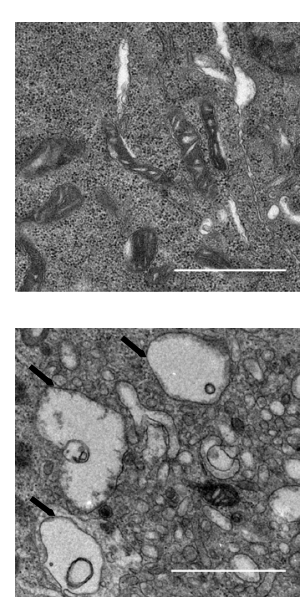

G
B

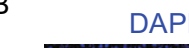

DAPI
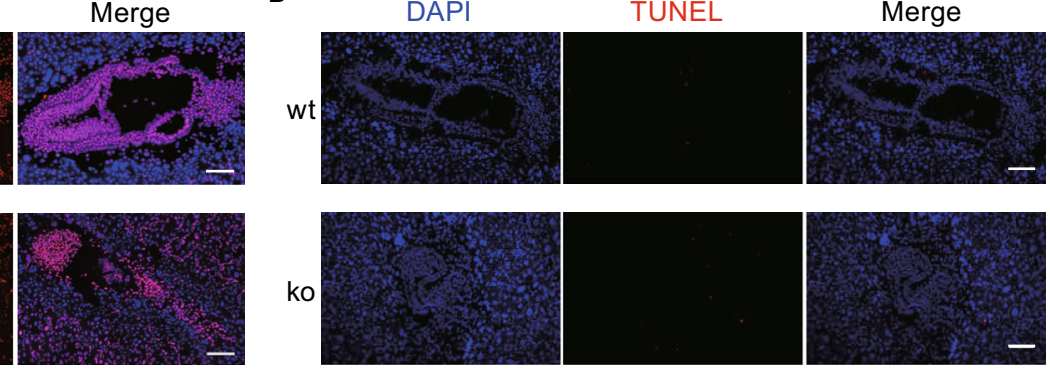

$40 x$

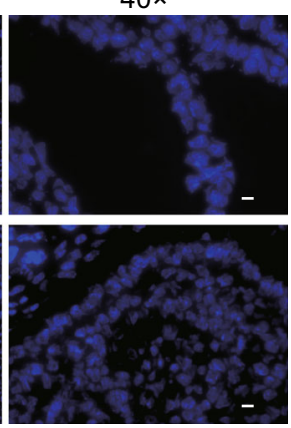

E
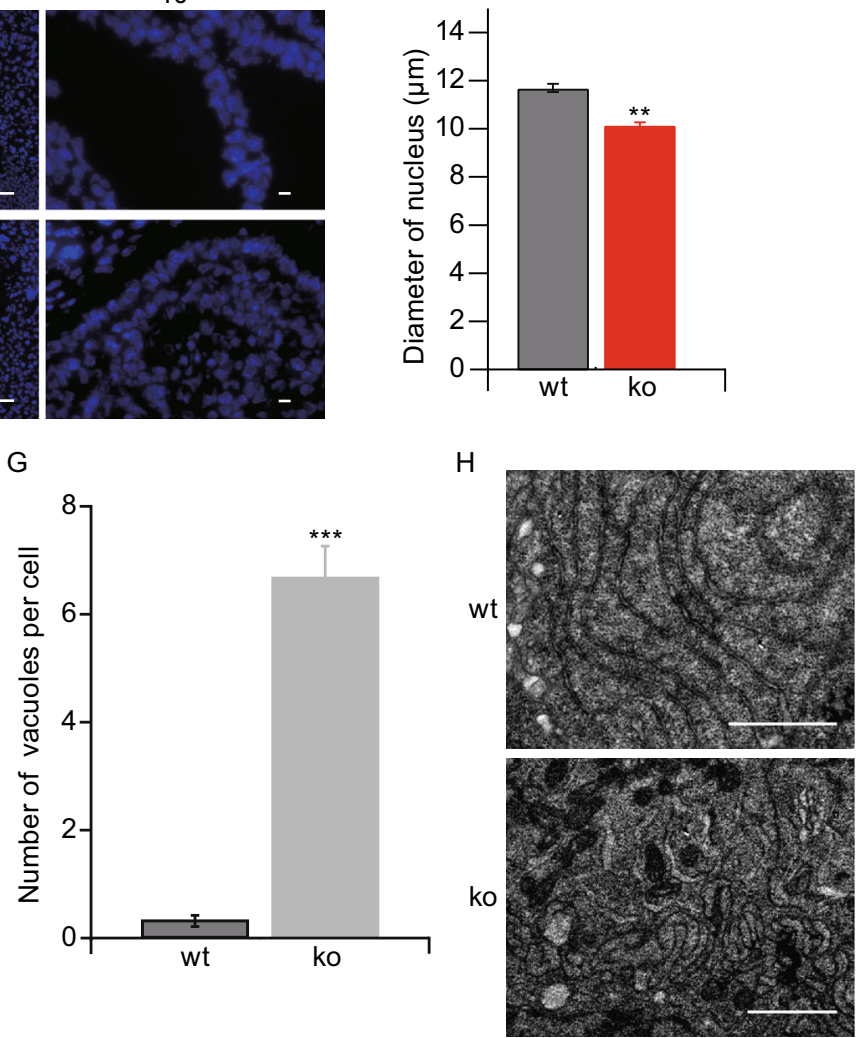

Figure 2. E7.5 Rab10 ${ }^{-1-}$ embryos are deficient in cell proliferation and show small nucleus, accumulated extensive vacuoles and hyperplasia ER. (A) Cell proliferation was assessed using Ki67 staining in E7.5 embryos from heterozygous intercrosses, scale bar $=100 \mu \mathrm{m}$. (B) Rab10-deficient embryos did not undergo apoptosis. A TUNEL assay was performed for apoptosis detection using an in situ cell death detection kit (Beyotime). (C) Quantification of Ki67-positive cells in wild-type and mutant embryos. Values are shown as the average \pm standard deviation, and the $P$-value was determined using a $t$-test. (D) Nuclear staining with DAPI of embryos

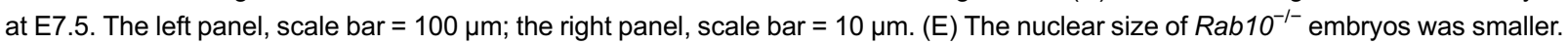

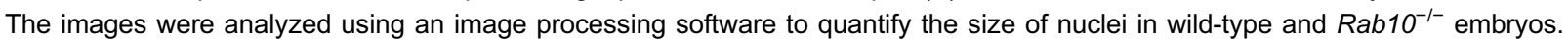
(F) The ultrastructure of isolated E7.5 embryos was stained and visualized using electron microscopy. Black arrowheads indicate vacuoles. Scale bar $=1 \mu \mathrm{m}$. (G) Quantification of vacuoles in wild-type and mutant embryos. Values are expressed as the average \pm standard deviation and the $P$-value was determined by a $t$-test. $(\mathrm{H})$ The ER exhibited hyperplasia in $R a b 10^{-1-}$ embryos. Total embryonic cells were analyzed in A and B; In (D), 4-5 parts of every embryo were randomly selected to calculate the size of nucleus; For $(F)$ and $(H)$, the cells were from epiblast.

severity of the phenotype, conditional knockout strategy is required for further clarification of Rab10's function. As Rab10 is expressed in many tissues, including lymphoid organs, liver, testis, ovary, etc. (BioGPS), tissue-specific knockout mouse models are needed to further define its physiological functions at different tissues. 


\section{FOOTNOTES}

This work was supported by grants from the National Natural Science Foundation (Grant No. 31270884), the Beijing Natural Science Foundation (5122026), and Youth Innovation Promotion Association, Chinese Academy of Sciences.

Pingping Lv, Yi Sheng, Zhenao Zhao, Wei Zhao, Lusheng Gu, Tao Xu and Eli Song declare that they have no conflict of interest.

All institutional and national guidance for the care and use of laboratory animals were followed.

Pingping Lv ${ }^{1,2}$, Yi Sheng ${ }^{1,3}$, Zhenao Zhao ${ }^{4}$, Wei Zhao ${ }^{1,2}$, Lusheng $\mathrm{Gu}^{1,3}$, Tao Xu ${ }^{1 凶}$, Eli Song ${ }^{1 凶}$

${ }^{1}$ National Laboratory of Biomacromolecules, Institute of Biophysics, Chinese Academy of Sciences, Beijing 100101, China

2 University of Chinese Academy of Sciences, Beijing 100049, China

${ }^{3}$ College of Life Science and Technology, Huazhong University of Science and Technology, Wuhan 430074, China

${ }^{4}$ State Key Laboratory of Reproductive Biology, Institute of Zoology, Chinese Academy of Sciences, Beijing 100101, China

$\checkmark$ Correspondence: xutao@ibp.ac.cn (T. Xu), songali@moon.ibp. ac.cn (E. Song)

\section{OPEN ACCESS}

This article is distributed under the terms of the Creative Commons Attribution License which permits any use, distribution, and reproduction in any medium, provided the original author(s) and the source are credited.

\section{REFERENCES}

Babbey CM, Ahktar N, Wang E, Chen CCH, Grant BD, Dunn KW (2006) Rab10 regulates membrane transport through early endosomes of polarized Madin-Darby canine kidney cells. Mol Biol Cell 17:3156-3175

Chen CC, Schweinsberg PJ, Vashist S, Mareiniss DP, Lambie EJ, Grant BD (2006) RAB-10 is required for endocytic recycling in the Caenorhabditis elegans intestine. Mol Biol Cell 17:1286-1297
Chen Y, Wang Y, Zhang J, Deng Y, Jiang L, Song E, Wu XS, Hammer JA, Xu T, Lippincott-Schwartz J (2012) Rab10 and myosin-Va mediate insulin-stimulated GLUT4 storage vesicle translocation in adipocytes. J Cell Biol 198:545-560

Chen S, Li L, Li J, Liu B, Zhu X, Zheng L, Zhang R, Xu T (2014) SEC-10 and RAB-10 coordinate basolateral recycling of clathrinindependent cargo through endosomal tubules in Caenorhabditis elegans. Proc Natl Acad Sci USA 111:15432-15437

Fürthauer M, González-Gaitán M (2009) Endocytic regulation of notch signalling during development. Traffic 10:792-802

Furthauer M, Gonzalez-Gaitan M (2009) Tales of 1001 functions: the multiple roles of membrane trafficking in development. Traffic 10:781-782

Kolotuev I, Apaydin A, Labouesse M (2009) Secretion of Hedgehogrelated peptides and WNT during caenorhabditis elegans development. Traffic 10:803-810

Lebreton S, Boissel L, Moreau J (2003) Control of embryonic Xenopus morphogenesis by a Ral-GDS/Xral branch of the Ras signalling pathway. J Cell Sci 116:4651-4662

Lerner DW, McCoy D, Isabella AJ, Mahowald AP, Gerlach GF, Chaudhry TA, Horne-Badovinac S (2013) A Rab10-dependent mechanism for polarized basement membrane secretion during organ morphogenesis. Dev Cell 24:159-168

Lučin P, Mahmutefendić H, Blagojević Zagorac G, llić Tomaš M (2014) Cytomegalovirus immune evasion by perturbation of endosomal trafficking. Cell Mol Immunol 12:154-169

Richter S, Voss U, Jurgens G (2009) Post-Golgi traffic in plants. Traffic 10:819-828

Stenmark H (2009) Rab GTPases as coordinators of vesicle traffic. Nat Rev Mol Cell Bio 10:513-525

Ulrich F, Heisenberg CP (2009) Trafficking and cell migration. Traffic 10:811-818

Xu XH, Deng CY, Liu Y, He M, Peng J, Wang T, Yuan L, Zheng ZS, Blackshear PJ, Luo ZG (2014) MARCKS regulates membrane targeting of Rab10 vesicles to promote axon development. Cell Res 24:576-594

Zerial M, McBride H (2001) Rab proteins as membrane organizers. Nat Rev Mol Cell Bio 2:107-117

Pingping Lv and Yi Sheng have contributed equally to this work. 